ORIGINAL

\title{
Behavioral and psychological symptoms of dementia (BPSD) and care burden : Examination in the facility staff for elderly residents
}

\author{
Naomi Kameoka1, Satsuki Sumitani², and Tetsuro Ohmori ${ }^{3}$ \\ ${ }^{1}$ Department of Psychiatry, Tokushima University Hospital, Tokushima, Japan, ${ }^{2}$ Academic support office for students with special needs, \\ Tokushima University, Tokushima, Japan, ${ }^{3}$ Department of Psychiatry, Graduate School of Biomedical Sciences, Tokushima University, \\ Tokushima, Japan
}

\begin{abstract}
Purpose : We investigated the cognitive function, behavioral and psychological symptoms of dementia (BPSD), and activities of daily living (ADLs) of elderly individuals admitted in care facilities. Moreover, the factors affecting the care burden experienced by facility staffs were examined. Method: 24 care facilities for elderly individuals participated in the study. The Revised Hasegawa Dementia Scale (HDS-R), Japanese version of the Neuropsychiatric Inventory (NPI), and Crichton Geriatric Behavioral Rating Scale (CGBRS) were used to evaluate cognitive function, BPSD, and ADL, respectively. The short Japanese version of the Zarit Burden Interview was used to assess the care burden. A multiple regression analysis was conducted with data obtained from 464 elderly individuals who fulfilled all the scales. Results: The care burden was correlated to the scores of HDS-R, but not with those of dysphoria/depression and disinhibition of NPI, restlessness of CGBRS, and subjective mood of CGBRS $\left(\mathrm{R}^{2}=0.309, \mathrm{p}<0.005\right)$. Conclusion : Dysphoria/depression, disinhibition, restlessness, and subjective mood, but not cognitive decline, have an effect on the care burden experienced by facility staffs who manage elderly individuals. These results indicated that the appropriate diagnosis and treatment of BPSD are important in reducing the burden of facility staffs. J. Med. Invest. 67: 236-239, August, 2020
\end{abstract}

Keywords : behavioral and psychological symptoms of dementia (BPSD), care burden, facility staff

\section{INTRODUCTION}

In developed countries, the rate of aging (percentage of individuals aged over 65 years in the total population) is increasing $(1,2)$. Japan has the highest rate of aging among all developed countries, and the rate can reach $30 \%$ in 2025 (3). The number of elderly individuals who require care is also increasing. Although the national policy includes the promotion of home care, supporting elderly individuals requiring care at home is challenging. According to the data obtained from the Ministry of Health, Labour and Welfare in 2016, the proportion of households in which elderly individuals aged over 65 years who require care and are cared for by people aged over 65 years has reached $54.7 \%$ (4). In actual settings, choosing an appropriate care facility is important, and the environment of facilities must be improved to help families leave their relatives without any worry.

Several elderly patients who are admitted in care facilities present with dementia, and care is an important part of nursing. A research about the burden of care experienced by the families of patients with dementia at home has revealed that the behavioral and psychological symptoms of dementia (BPSD) affect the burden of care rather than the severity of the core symptoms of dementia, such as memory impairment and disorientation (5). However, the factors affecting the burden of care experienced by facility staffs who manage elderly individuals with dementia have not been fully elucidated. Thus, we investigated the cognitive function, BPSD, and activities of daily living (ADLs)

Received for publication December 3, 2019; accepted February 18, 2020.

Address correspondence and reprint requests to Naomi Kameoka, Department of Psychiatry, Tokushima University Hospital, Tokushima, 2-50-1, Kuramoto-cho, Tokushima 770-8503, Japan and Fax : +81-633-7131. of elderly individuals admitted in care facilities. Moreover, the factors affecting the burden of care experienced by care facility staffs were examined.

\section{PATIENTS AND METHODS}

\section{Subject}

In total, 24 facilities for elderly individuals from the Social Welfare Corporation Kenshokai Group participated in the study. The written research protocol of the current study was explained to 1,571 residents and their families from July to August 2011 , and they were asked to take part in the study. The survey was conducted on 669 individuals who consented to participate. The data obtained from 464 elderly individuals (103 men, 361 women ; mean age : $86.27 \pm 6.96$ years) who fulfilled all the scales were included in the analysis. The nurse or caregiver in charge of the relevant resident answered the question.

The ethics committee of Tokushima University approved the study.

\section{Measures}

We investigated the cognitive function, BPSD, and ADL of the residents and the burden of care experienced by the facility staffs. The Revised Hasegawa dementia scale (HDS-R) (6), Japanese version of the Neuropsychiatric Inventory (NPI) (7), Crichton Geriatric Behavioral Rating Scale (CGBRS) (8) were used to evaluate cognitive function, severity and frequency of BPSD, behavior and activities, including ADL, respectively.

HDS-R is a cognitive function evaluation scale commonly used in Japan. It comprises nine items, namely : age, orientation to time, orientation to place, repeating three words, serial 7s, numbers from reverse, recall of three words, recall of five objects, and providing names of vegetables (verbal fluency). The evaluation is 
based on a 30-point scale, and a score of 20 or less indicates a risk of dementia. A positive correlation between Mini-mental State Examination score and total score of HDS-R was observed (6).

NPI comprises 10 items, which are as follows : delusion, hallucination, agitation/aggression, dysphoria/depression, anxiety, euphoria/elation, apathy/indifference, disinhibition, irritability/ lability, and aberrant motor behavior. Severity (0-3) and frequency (0-4) must be assessed, and the results are assigned with a score (7).

The CGBRS is composed of 11 items, which are as follows : mobility, orientation, communication, cooperation, restlessness, dressing, feeding, continence, sleep, objective mood, and subjective mood. Severity is assessed by assigning scores ranging from 1 to 5 (8). Objective mood is an item used for evaluating objective instability. A score of 1 is assigned when the mood is good with vivid facial expressions and 5 when the mood is unstable with delusion and hallucination. Subjective mood is used to evaluate depression and hypochondria. A score of 1 is assigned when the mood is good with subjective feelings of well-being or euphoria and 5 when the mood is bad with suicidal or death wishes, mutism, or agitation to the point of incoherence.

The short Japanese version of the Zarit Burden Interview (J-ZBI_8) was used to assess burden of care (Table 1) $(9,10)$. J-ZBI_8 is composed of eight items from the 22 question items that comprise the J-ZBI, which include five personal strains (degree of negative feelings for situations requiring nursing care) and three items for role strain (degrees of nursing care hindering social life). Each item is rated from 0 (never) to 4 (nearly always). Since the participants of this survey were facility staffs working in nursing homes, role strain was not evaluated, and the total score for the five items of personal strain (hereinafter referred to as J-ZBI_8-PS) was calculated.

\section{Analysis}

First, the total J-ZBI_8-PS, HDS-R, NPI, and CGBRS scores were used to examine the correlation between the degree of burden of care and cognitive decline as well as overall behavior, in cluding BPSD and ADL. Next, the correlation between the total J-ZBI_8-PS score and the score of each item in NPI and CGBRS was examined. A multiple regression analysis was performed using items with significant correlations and absolute correlation coefficients of 0.2 or higher as independent variables and total J-ZBI_8-PS scores as dependent variables. The Statistical Package for the Social Sciences software version 14.0 for Windows was used, and a $\mathrm{p}$ value $<0.05$ was considered statistically significant.

\section{RESULTS}

Table 2 shows the correlation between the total HDS-R, NPI, and CGBRS scores and J-ZBI_8-PS score. The total J-ZBI_8-PS score had a weak negative correlation to the total HDS-R score $(\mathrm{r}=-0.285, \mathrm{p}<0.05)$. Meanwhile, a weak positive correlation $(\mathrm{r}$ $=0.397, p<0.05)$ was observed between the total J-ZBI_8-PS score and the total NPI score, and the total J-ZBI_8-PS score had a slight positive correlation $(r=0.412, p<0.05)$ to the total CGBRS score.

Table 2. Correlation between the total score of HDS-R, NPI or CGBRS and J-ZBI 8-PS

\begin{tabular}{lllll}
\hline & & HDS-R & NPI score & CGBRS score \\
\hline J-ZBI_8-PS & $\mathrm{r}$ & -.285 & .397 & .412 \\
& $\mathrm{p}$ & .000 & .000 & .000 \\
HDS-R & $\mathrm{r}$ & 1 & -.270 & -.416 \\
& $\mathrm{p}$ & & .000 & .000 \\
NPI score & $\mathrm{r}$ & -.270 & 1 & .487 \\
& $\mathrm{p}$ & .000 & & .000 \\
\hline
\end{tabular}

Table 3 shows the correlation between the NPI and CGBRS items and the J-ZBI_8-PS score. The total J-ZBI_8-PS score had a significant correlation to all items, except mobility. Among the NPI items used to evaluate BPSD, delusion, agitation/aggression, dysphoria/depression, anxiety, disinhibition, irritability/lability, and aberrant motor behavior had a correlation coefficient of 0.2 or higher. Among the CGBRS items used to assess ADL, orientation, communication, cooperation, restlessness, dressing, continence, objective mood, and subjective mood had a correlation coefficient of 0.2 or higher.

A multiple regression analysis was performed using 7 of 10 NPI items and 8 of 11 CGBRS items with correlation coefficients of 0.2 or higher, which were used as independent variables, and results showed that the J-ZBI_8-PS score was significantly correlated to dysphoria/depression of NPI, disinhibition of NPI, restlessness of CGBRS, and subjective mood of CGBRS $\left(\mathrm{R}^{2}=\right.$ $0.309, \mathrm{p}<0.005)$ (Table 4).

Table 1. A short version of the Japanese version of Zarit caregiver burden interview (J-ZBI_8). In this study, "your relative" in the original version was translated into "subject you care".

【Personal strain】

- Do you feel embarrassed over your relative's behavior (behavior of subject you care)?

- Do you feel angry when you are around your relative (subject you care)?

- Do you feel strained when you are around your relative (subject you care)?

- Do you wish you could leave the care of your relative (subject you care) to someone else?

- Do you feel uncertain about what to do about your relative (subject you care)?

【Role strain】

- Do you feel that your relative currently affects your relationships with other family members or friends in a negative way?

- Do you feel that your social life has suffered because you are caring for your relative?

- Do you feel uncomfortable about having friends over because of your relative? 
Table 3. Correlation between each item of NPI or CGBRS and care burden (J-ZBI_8-PS)

\begin{tabular}{|c|c|c|c|}
\hline & & \multicolumn{2}{|c|}{ J-ZBI_8-PS } \\
\hline & & $\mathrm{r}$ & $\mathrm{p}$ \\
\hline \multirow[t]{10}{*}{ NPI } & Hallucination & .159 & .001 \\
\hline & Delusion & .208 & .000 \\
\hline & Agitation/Aggression & .348 & .000 \\
\hline & Dysphoria/Depression & .252 & .000 \\
\hline & Anxiety & .201 & .000 \\
\hline & Euphoria/Elation & .183 & .000 \\
\hline & Apathy/Indifference & .066 & .009 \\
\hline & Disinhibition & .315 & .000 \\
\hline & Irritability/Lability & .355 & .000 \\
\hline & Aberrant motor behavior & .296 & .000 \\
\hline \multirow[t]{11}{*}{ CGBRS } & Mobility & .041 & .378 \\
\hline & Orientation & .281 & .000 \\
\hline & Communication & .295 & .000 \\
\hline & Co-operation & .308 & .000 \\
\hline & Restlessness & .396 & .000 \\
\hline & Dressing & .225 & .000 \\
\hline & Feeding & .143 & .000 \\
\hline & Continence & .220 & .000 \\
\hline & Sleep & .150 & .001 \\
\hline & Objective mood & .333 & .000 \\
\hline & Subjective mood & .335 & .000 \\
\hline
\end{tabular}

\section{DISCUSSION}

Dysphoria/depression, disinhibition, restlessness, and subjective mood affect the burden of care experienced by care facility staffs. The severity of the core symptoms of dementia does not affect the burden of care. In addition, physical problems, such as mobility, dressing, feeding, and continence, did not have any effect. BPSD causes a higher burden than the core symptoms of dementia or the physical problems of the person cared for by the facility staff who has a certain skill in ADL assistance. The impact of BPSD on burden of care is consistent with that in a study conducted on family caregivers (5).

Some studies have assessed the burden of care experienced by care facility staffs. However, the results were contrasting. Song (11) has shown that the factors that increase the burden include agitation, aggression, delusion, hallucination, aberrant motor behavior, and disinhibition. Moreover, Zwijsem (12) has reported that agitation and aggression, followed by disinhibition and irritability/lability, cause the highest burden. Miyamoto (13) has shown that impaired function, female sex, aggressive and other types of inappropriate behaviors (quarrelling and screaming) are the patient factors correlated to the burden experienced by formal caregivers. The varying results of previous studies may be attributed to the differences in survey target or measurement metrics. Fauth (14) concludes that symptoms revealed as "most problematic" vary tremendously, depending on whether the criterion used is symptom prevalence, intensity, caregiver distress, or associations with caregiver depressive symptoms.

Our results about disinhibition and restlessness are consistent with those of previous studies $(11,12)$, but a strong association with depression and the subjective mood was first reported in this study. Moreover, depressive mood, a common symptom among patients with dementia, required attention from the perspective of burden of care. Whether this result is a characteristic

Table 4. Factors affecting care burden

\begin{tabular}{llccc}
\hline & & Average & $\begin{array}{l}\text { Standardization } \\
\text { factor }\end{array}$ & Significance \\
\hline HDS-R & & $10.9 \pm 7.4$ & -0.103 & 0.051 \\
NPI & Delusion & $0.9 \pm 2.1$ & -0.095 & 0.056 \\
& Agitation/Aggression & $1.2 \pm 2.4$ & 0.12 & 0.847 \\
& Dysphoria/Depression & $0.6 \pm 1.5$ & 0.105 & $0.032^{*}$ \\
& Anxiety & $0.9 \pm 1.9$ & -0.017 & 0.727 \\
& Disinhibition & $0.7 \pm 2.0$ & 0.147 & $0.004^{*}$ \\
& Irritability/Lability & $1.1 \pm 2.3$ & 0.103 & 0.089 \\
& Aberrant motor behavior & $1.0 \pm 2.6$ & 0.050 & 0.308 \\
CGBRS & Orientation & $2.5 \pm 1.2$ & -0.007 & 0.911 \\
& Communication & $1.8 \pm 1.0$ & 0.098 & 0.089 \\
& Co-operation & $2.2 \pm 1.0$ & 0.074 & 0.124 \\
& Restlessness & $1.8 \pm 1.0$ & 0.159 & $0.003^{*}$ \\
& Dressing & $2.5 \pm 1.2$ & -0.023 & 0.670 \\
& Continence & $2.7 \pm 1.3$ & 0.091 & 0.066 \\
& Objective mood & $2.1 \pm 1.0$ & -0.013 & 0.819 \\
& Subjective mood & $1.8 \pm 1.0$ & 0.142 & $0.008^{*}$
\end{tabular}

A multiple regression analysis using the forced injection method was performed with 7 out of $10 \mathrm{NPI}$ items and 8 out of 11 CGBRS items with correlation coefficients of 0.2 or more as independent variables, and J-ZBI_8-PS as dependent variables. $\left(\mathrm{R}^{2}=0.309\right)$

$* \mathrm{P}<0.005$ 
of a care facility that manages individuals with dementia in Japan must be examined.

Similar to the study of Miyamoto et al. (13), we used personal strain, which is a subscale of J-ZBI_8, to evaluate the burden of care experienced by facility staffs (Table 1). ZBI has a two-factor structure of personal strain and role strain, and the factor structure is retained in the shortened version established by Whitlach et al. (15) and Bédard et al. (16) as well as in the short Japanese version of J-ZBI 8. The three items of role strain can be used to evaluate the degree of social hindrance caused by providing care and are intended for home caregivers. These items were excluded from the current survey because they do not reflect the burden experienced by facility staffs. Rather, the five items of personal strain were used to assess negative feelings about nursing care. These items are believed to reflect the burden of care that is not based on the caregiver's position and that is appropriate for this survey. In fact, in a cross-validation study of J-ZBI_8, the personal strain score was significantly correlated to the physical and mental fatigue experienced by caregivers (10).

Our study showed that a better understanding of BPSD and improvement in coping skills among facility staffs can improve the quality of care and contribute to reducing burden.

\section{LIMITATION}

Elderly residents, including those with and without dementia, were included in this survey. Several participants without a definitive diagnosis of dementia had an HDS-R of 20 points or less and were suspected of having a declined cognitive function. In addition, even when the patient had a definite diagnosis of dementia, the subtype diagnosis cannot be sufficiently evaluated.

\section{CONCLUSION}

The relationship between the psychological and behavioral symptoms of elderly individuals admitted in care facilities and the burden of care experienced by facility staffs were investigated. Results showed that the degree of burden was correlated to BPSD, and dysphoria/depression, disinhibition, restlessness, and subjective mood affected the burden of care experienced by staffs. Thus, the appropriate diagnosis and treatment of BPSD symptoms are important in reducing the burden experienced by care facility staffs.

\section{CONFLICTS}

This study was financially supported by and conducted with the cooperation of the social welfare corporation Kenshokai.

\section{REFERENCES}

1. World population prospects. The 2015 Revision (in Japanese). UN.

2. National census (in Japanese). Ministry of Internal Affairs and Communications.

3. National Institute of Population and Social Security Research. Population Projections for Japan : 2016-2065. Population Research Series 2017 (in Japanese)

4. National Life Basic Survey. (in Japanese) Ministry of Health, Labor and Welfare. 2016

5. Lee J, Bakker T, Duivenvoorden H, Dröes RM : Multivariate models of subjective caregiver burden in dementia : A systematic review. Ageing Research Reviews 15: 76-93, 2014

6. Hasegawa K : Evaluation scale of mental function in old age (in Japanese). Rinsho Seisin Yakuri 2 : 3-11, 1999

7. Hirono N, Mori E, Ikejiri Y, Imamura T, Shimomura T, Hashimoto M, Yamashita H, Ikeda M : Japanese version of the Neuropsychiatric Inventory -A scoring system for neuropsychiatric disturbances in dementia patients (in Japanese). BRAIN and NERVE 49(3) : 266-271, 1997

8. Endo H, Umemoto M, Satake S, Matsuyama Z, Miura $\mathrm{H}$ : Study for relationship between Crichton Geriatric Behavioral Rating Scale and caregiver burden in people with cognitive dysfunction (in Japanese). Ronen Seishin Igaku Zasshi 19:569-576, 2008

9. Arai Y, Tamiya N, Yano E: The short version of the Japanese version of the Zarit Caregiver Burden Interview (J-ZBI_8) : its reliability and validity (in Japanese). Nihon Ronen Igaku Zasshi 40 : 497-503, 2003

10. Kumamoto K, Arai Y, Ueda T, Washio M : Cross-validation of the short version of the Japanese version of the Zarit Caregiver Burden Interview (J-ZBI_8) (in Japanese). Nihon Ronen Igaku Zasshi 41 : 204-210, 2004

11. Song JA, Oh Y: The Association Between the Burden on Formal Caregivers and Behavioral and Psychological Symptoms of Dementia (BPSD) in Korean Elderly in Nursing Homes. Archives of Psychiatric Nursing 29 : 346-354, 2015

12. Zwijsen SA, Kabboord A, Eefsting JA, Hertogh CMPM, Pot AM, Gerritsen DL, Smalbrugge M : Nurses in distress? An explorative study into the relation between distress and individual neuropsychiatric symptoms of people with dementia in nursing homes. Int J Geriatr Psychiatry 29 : 384-391, 2014

13. Miyamoto $\mathrm{Y}$, Tachimori $\mathrm{H}$, Ito $\mathrm{H}$ : Formal caregiver burden in dementia : Impact of behavioral and psychological symptoms of dementia and activities of daily living. Geriatric Nursing 31(4) : 246-253, 2010

14. Fauth EB, Gibbons A : Which behavioral and psychological symptoms of dementia are the most problematic? Variability by prevalence, intensity, distress ratings, and associations with caregiver depressive symptoms. Int J Geriatr Psychiatry $29: 263-271,2014$

15. Whitlatch CJ, Zarit SH, von Eye A : Efficacy of interventions with caregivers : a reanalysis. Gerontologist 31(1) : 9-14, 1991

16. Bédard M, Molloy DW, Squire L, Dubois S, Lever JA, O'Donnell M : The Zarit Burden Interview : a new short version and screening version. Gerontologist 41(5) : 652-657, 2001 